\title{
Rapport à l'écrit et construction de connaissances disciplinaires. Étude de cas
}

\section{Marie-Cécile Guernier et Christine Barré-De Miniac}

\section{Q OpenEdition \\ 1 Journals}

\section{Édition électronique}

URL : http://journals.openedition.org/pratiques/1457

DOI : 10.4000/pratiques. 1457

ISSN : 2425-2042

Éditeur

Centre de recherche sur les médiations (CREM)

\section{Édition imprimée}

Date de publication : 15 décembre 2009

Pagination : 203-217

\section{Référence électronique}

Marie-Cécile Guernier et Christine Barré-De Miniac, «Rapport à l'écrit et construction de connaissances disciplinaires. Étude de cas », Pratiques [En ligne], 143-144 | 2009, mis en ligne le 13 juin 2014, consulté le 10 décembre 2020. URL : http://journals.openedition.org/pratiques/1457 ; DOI : https://doi.org/10.4000/pratiques.1457

\section{(c) Tous droits réservés}




\title{
Rapport à l'écrit et construction de connaissances disciplinaires. Étude de cas
}

\author{
Marie-Cécile Guernier \\ Université Claude Bernard Lyon 1 - \\ IUFM, LIDILEM Université Stendhal Grenoble 3 \\ Christine Barré-De Miniac \\ Université Joseph Fourrier Grenoble 1 - \\ IUFM, LIDILEM Université Stendhal Grenoble 3
}

\section{1. Écrits disciplinaires : apprentissages et rapport à l'écrit}

\section{1. Écrire pour apprendre : diversité et normalisation}

Écrire permettant d'apprendre, une des fonctions principales des activités d'écriture en classe consiste à consigner et formaliser les savoirs en cours de construction, à retenir, à réviser et à restituer le plus souvent dans le cadre des évaluations. Ainsi l'activité d'écriture est-elle au cœur de l'activité épistémique et de conceptualisation.

L'école ayant organisé l'enseignement des savoirs selon des disciplines le plus souvent issues des disciplines universitaires, chaque discipline scolaire a développé des modalités et des formes d'écriture spécifiques, en lien d'une part avec les habitudes et normes scolaires, d'autre part avec les savoirs disciplinaires à construire et ses propres conceptions épistémologiques.

De ce fait, les fonctions pédagogique et didactique assignées aux écrits et aux activités d'écriture entraînent leur transformation et leur adaptation au projet et au contexte d'enseignement et d'apprentissage dans lesquels ils sont utilisés : objectifs d'apprentissage visés, niveau d'enseignement, situation d'enseignement-apprentissage. Les écrits scolaires présentent donc des caractéristiques particulières. Et chaque discipline scolaire a ainsi développé des normes qui lui sont propres. C'est ainsi que la dissertation est un exercice d'écriture réservé au lycée et plutôt aux disciplines des sciences humaines (français, philosophie, histoire, géographie, langues), alors qu'au collège on écrira plutôt des rédactions. On lit et écrit des récits plutôt en cours de français et des textes expositifs, informatifs et explicatifs plutôt en biologie, histoire, géographie. L'inverse est pourtant possible. En mathématique, on lit des problèmes et des énoncés d'exercices et on rédige des dé- 
monstrations et des résultats. Alors qu'en grammaire on fait des exercices mais on ne résout pas de problèmes. Alors qu'on pourrait le faire. Chaque discipline développe ainsi des formes discursives spécifiques : consigne, questions, énoncé de problème, énoncé d'exercices, texte didactique qui présente une notion, réponses types, exercices d'écriture types, etc.

La norme scripturale scolaire peut donc être envisagée selon deux aspects : premièrement une norme encadrante générale qui concerne d'une part les aspects pragmatiques, c'est-à-dire les formes et les types d'usages de l'écrit en contexte scolaire, d'autre part les aspects linguistiques et codiques de l'écrit : correction grammaticale et orthographique, registre en conformité avec les usages scolaires ; deuxièmement une norme spécifique qui concerne les genres textuels et discursifs en usage dans chaque discipline en lien avec les exercices et les types de textes développés par chaque discipline (Barré-De Miniac \& Reuter, 2006) et que l'on peut apparenter aux genres secondaires décrits par Bakhtine (Bakhtine, 1984).

L'élève se trouve ainsi confronté à une diversité scripturale importante et doit identifier ce qui caractérise les spécificités des écrits et des activités d'écriture de chaque discipline et en apprendre les modalités pour apprendre les savoirs mêmes de la discipline (Lahanier-Reuter \& Reuter, 2006). Ainsi apprendre à écrire à l'école ou écrire pour apprendre à l'école consiste-t-il à apprendre à écrire selon les normes et les règles scolaires, et apprendre les savoirs d'une discipline consiste-til aussi à apprendre les modalités et les normes scripturales de la discipline.

Ces différents aspects ont été déjà largement décrits (INRP, 1999, Pratiques, 2002a et b), et selon une perspective sociologique / socioculturelle, l'hypothèse a été formulée (Charlot, Bautier \& Rochex, 1992, Charlot, 1999, Lahire, 1993) que ces types d'usages et ces formes discursives normalisés, parce qu'ils sont spécifiques à l'école et construisent d'une part un rapport distancié entre le langage et ses référents, d'autre part une conception objectivante du langage pouvaient en partie être responsables des difficultés scolaires de certains élèves, et plus particulièrement de ceux qui les maîtrisaient le moins. En revanche, on sait moins comment les élèves perçoivent ces normes et comment ils se les approprient d'un point de vue psycho-cognitif. C'est ce point de vue que nous voudrions explorer ici à partir de la notion de rapport à l'écrit à propos d'activités d'écriture proposées dans trois disciplines : le français, l'histoire, les mathématiques, et une activité transversale : un atelier d'écriture.

\subsection{Rapport à l'écrit et didactique}

En effet, si il est indéniable que le décalage entre les pratiques personnelles inscrites dans les pratiques du groupe social et culturel auquel appartient l'élève et les pratiques scolaires puisse constituer un obstacle à l'apprentissage de l'écrit scolaire pour certains élèves, il apparaît aussi que de nombreux élèves issus de groupes sociaux et culturels réputés en décalage avec les pratiques langagières scolaires parviennent à maîtriser les formes scolaires de l'écrit (Lahire, 1995). On peut donc faire l'hypothèse que des facteurs d'un autre ordre sont à prendre en compte. Le rapport à l'écrit nous semble doté d'un fort pouvoir heuristique (Delamotte Ret al., 2000, Chartrand (dir.), 2008).

La notion de rapport à l'écrit permet d'explorer les fondements psycho-cognitifs de l'investissement d'un individu dans l'activité scripturale. En effet, lire et écrire ne se réduisent pas à des techniques mais s'inscrivent dans une dynamique personnelle et intime dans laquelle c'est l'individu tout entier qui est concerné.

La notion de rapport à l'écrit recouvre « l'ensemble des significations construi- 
tes par le scripteur à propos de l'écriture, de son apprentissage et de ses usages. Significations singulières pour les uns, partagées par le groupe social pour d'autres, le groupe culturel pour d'autres encore. L'ensemble étant de toutes manières retravaillé, réorganisé par un sujet unique, ce que désigne le singulier de l'expression rapport à. ") (Barré-De Miniac, 2000 et 2002). Le rapport à l'écriture d'un sujet, que nous utiliserons ici comme outil d'analyse, s'appuie sur le modèle en quatre dimensions proposé par Christine Barré-De Miniac :

1. L'investissement du sujet dans les activités d'écriture tant au niveau affectif que quantitatif. Cette notion réfère à la force de l'attachement et à l'intérêt pour l'écriture. Cette force peut être orientée soit positivement soit négativement (par exemple rejet) et d'intensité diverse : forte, moyenne, ou faible.

2. Les opinions, sentiments et valeurs attribués à l'écriture et à ses usages; et les attitudes qui en découlent.

3. Les conceptions et les représentations concernant l'activité d'écriture et son apprentissage.

4. Les modes de verbalisation sur l'écrit, l'écriture et l'activité scripturale telle que le sujet la pratique.

Ainsi au-delà d'une explication des comportements des élèves face à l'écrit par le biais de la motivation personnelle et / ou de la déficience cognitive, la notion de rapport à l'écrit permet de s'intéresser à d'autres paramètres mobilisés par le scripteur et plus particulièrement ceux qui concernent la signification qu'il accorde à l'activité scripturale. La notion de rapport à apporte des éclairages qui peuvent nous aider à mieux cerner ce qui se trame entre le sujet et l'écrit auquel il est confronté. Elle peut donc constituer un éclairage pertinent pour analyser comment les élèves perçoivent l'activité scripturale dans le cadre scolaire et plus particulièrement comment ils perçoivent les normes associées aux écrits scolaires en général et selon les disciplines en particulier. Elle permet aussi de mesurer quelle appréciation de cette normalisation les élèves construisent, en positif ou en négatif, et par rapport à leur propre conception de l'écrit ; comment les élèves s'adaptent aux exigences et règles imposées par l'école ; comment ils en tirent profit ; quelle imbrication ils ont construite entre leur conception personnelle de l'écrit et la normalisation scolaire de l'écrit; et quels sont les effets sur l'apprentissage.

Nous cherchons donc à relier les trois pôles de l'activité scripturale quand elle est mise en œuvre dans une discipline scolaire :

- construction de connaissances : dimension épistémique,

- normalisation scripturale : dimension sociale

- rapport à l'écrit : dimension psychologique

Dans la mesure où la notion de rapport à l'écrit s'inscrit dans une perspective subjective, nous illustrerons ici le pouvoir heuristique de cet outil par l'étude du cas de Céline. Ce choix est fondé par notre projet de recherche qui, parce qu'il consiste à mieux appréhender ce qui relève de l'individuel dans le processus d'apprentissage, impose comme nécessaire, à certaines étapes de l'exploration, de partir de l'individu. Par ailleurs, il nous semble que s'agissant de didactique, ce choix constitue une étape incontournable, dans la mesure où un professeur travaille avec des sujets sur lesquels il cherche à agir pour qu'ils apprennent et s'engagent personnellement dans un processus de transformation intellectuelle et épistémique. Ainsi le triangle didactique est-il toujours reconfiguré par l'individu, élève ou professeur. De ce fait, il nous semble nécessaire, avant d'essayer de dégager des profils plus généraux, de porter notre attention sur des sujets précis et singuliers. 


\section{Céline : scolarité chaotique et rapport à l'écrit positif}

\subsection{Trajectoire scolaire de Céline}

Nous nous intéresserons donc ici à Céline, âgée d'une vingtaine d'années, élève en classe de première littéraire au Collège et Lycée Élitaire Pour Tous (CLEPT) de l'Académie de Grenoble, qui scolarise les élèves décrocheurs. Dans l'entretien qu'elle nous accorde, Céline raconte qu'après une scolarité sans problème à l'école élémentaire, les choses se gâtent pour elle à partir de la classe de quatrième. A l'issue de la classe de troisième, elle est orientée en BEP, puis comme elle y réussit bien et parce que les études de secrétariat ne l'intéressent pas, elle demande à réintégrer le cursus général au niveau de la classe de seconde. Là, elle constate que ce qu'elle a appris dans la voie professionnelle est en décalage avec les bases requises pour suivre en seconde. Elle travaille alors beaucoup pour rattraper son retard et est admise en classe de première. Mais de nouveau Céline éprouve des difficultés à suivre et nous dit qu'elle se sent peu aidée par les professeurs. Elle quitte donc le lycée en cours d'année, et comme elle a 18 ans, se met à travailler comme caissière dans un supermarché. Elle constate très vite que ce n'est pas un métier pour elle et qu'elle a d'autres aspirations. Après deux années d'interruption, elle décide donc de reprendre le chemin de l'école, élabore un dossier pour être admise au "lycée des décrocheurs », passe les entretiens et est admise en classe de seconde. Quand nous la rencontrons, elle est en classe de première littéraire. C'est une élève assidue qui veut avant tout réussir son baccalauréat. Elle a raccroché.

Le parcours de Céline n'est pas complètement banal, sans être exceptionnel non plus. C'est en raison de ce parcours que Céline, et ses camarades de classe qui ont tous des parcours à peu près similaires, nous ont intéressées. En effet, elle est une élève volontaire : ce qui exclut la thèse du manque de motivation. De plus, c'est une élève qui réussit, et depuis notre rencontre, elle a réussi au baccalauréat sans difficulté : ce qui exclut la thèse de la déficience intellectuelle et celle de la désocialisation. Au cours des deux longs entretiens semi-directifs que nous avons avec Céline, nous avons pu constater qu'elle a une bonne maîtrise de la langue : ce qui exclut la thèse des lacunes langagières. Céline est donc une jeune fille ordinaire qui cependant a failli quitter le système scolaire sans diplôme et qui était engagée dans la voie de l'exclusion scolaire, puis peut-être professionnelle. Pour ces différentes raisons, elle nous a paru être en mesure, en explicitant comment elle travaille en classe, de procéder à un retour méta-réflexif sur ce qu'elle fait, de formuler ses conceptions personnelles de l'écriture, de décrire les activités d'écriture menées en classe dans les différentes disciplines et d'expliquer comment elle apprend à partir de ce travail d'écriture.

\subsection{Un rapport positif à l'écrit scolaire et extra scolaire}

Commençons tout d'abord par remarquer que, dans le discours qu'elle construit au cours de l'entretien, Céline décrit un rapport positif à l'écrit scolaire et extra scolaire. Elle apprécie l'écriture et écrire. Pour le mettre en évidence, nous utilisons les quatre dimensions décrites ci-dessus :

- l'investissement, c'est-à-dire l'intérêt effectif de Céline pour l'écrit et la quantité d'énergie qu'elle y consacre,

- les opinions, sentiments et valeurs qu'elle accorde à l'écriture et à ses usages,

- les conceptions, c'est-à-dire la manière dont elle se représente l'activité d'écriture et son apprentissage, 
- les modes de verbalisations, c'est-à-dire la manière dont elle parle de l'écriture et de son activité scripturale.

Le rapport positif à l'écrit de Céline s'exprime d'emblée explicitement :

\section{Extrait 1}

Céline : J'aime bien écrire franchement pour moi l'écriture c'est une des plus belles inventions avec la musique

Enquêteur: Qu'est-ce que tu aimes bien dans l'écriture

Céline : C'est beau je veux dire l'écriture c'est les mots je trouve la littérature c'est un peu de l'architecture c'est une construction de mots suivant que l'on emploie ce mot cela change de sens il y a plein de nuances si on l'a mis là ce n'est pas pour rien et puis les mots c'est beau

Enquêteur: Et tu écris toi même

Céline : Je brouillonne je fais des petits brouillons je gribouille quoi [...] c'est vraiment pour dire n'importe quoi mais peut-être tous les jours j'écris deux trois petites phrases voilà c'est plus comme ça et des fois je me lance bon après c'est pour moi mais bon j'aime bien [...] des fois je marche dans la rue il y a une idée qui me vient n'importe quoi je la mets dans un petit coin après je verrai ce que j'en ferai plus tard après il y a un moment où d'un coup tous les petits mots que j'ai mis au fil du temps je vois un lien entre eux et je les rassemble pour écrire un petit texte voilà

L'investissement de Céline dans l'écriture est fort. Écrire est chez elle une pratique personnelle fréquente. Les opinions et sentiments qu'elle exprime à propos de l'écrit s'inscrivent dans les ordres de l'esthétique et du sensible. Le discours est valorisant. Le mode de verbalisation est explicite : Céline est capable de décrire son activité d'écriture de façon précise. Par ailleurs, son discours développe une conception élaborée de l'écrit, construite à partir de l'analyse des écrits des écrivains, c'est-à-dire d'un modèle d'écriture que l'on peut qualifier d'experte.

\section{Extrait 2}

[...] les écrivains je me dis mettre déjà sur papier leurs idées comme ça leurs pensées que ce soit structuré nous transmettre un message nous faire passer quelque chose enfin je veux dire déjà moi écrire dix lignes c'est dur mais alors écrire un livre en entier parler de ces choses là qui sont toujours d'actualité en plus voilà je trouve ça beau tout ce qui est littéraire et tout cela permet un peu de s'évader un petit peu oui c'est déjà subjectif quoi je trouve

Dans cet extrait, Céline insiste sur la complexité de l'élaboration du texte écrit en construisant une dialectique entre structurer une pensée et transmettre un message, c'est-à-dire en reliant la dimension introspective de l'écriture (trouver les mots qui expriment ce que l'on pense) et la dimension extravertie (trouver les mots pour l'autre), entre se dire et dire à l'autre. Ce faisant, elle établit une distinction précise ente l'oral et l'écrit.

\section{Extrait 3}

je pense que d'écrire cela permet de mieux structurer sa pensée parce que de l'avoir dans la tête comme ça et de le dire à l'oral on utilise des mots qui ne passent pas forcément à l'écrit et puis c'est vague on voit la réaction de la personne à qui on s'adresse et puis ah non ce n'est peut-être pas trop ça et puis on bifurque tandis qu'à l'écrit bon sur la feuille on est vraiment obligé de construire un raisonnement il n'y a pas quelqu'un on ne voit pas la réaction en face donc on se confronte plus à soi même je pense avec l'écrit [...] à l'oral on va se confronter vers quelqu'un tandis $q u$ 'à l'écrit on est seul face à sa copie on réfléchit on se pose on se répond à soimême quoi est-ce que je pense ça comment je vais le formuler comment je vais le développer est-ce que c'est essentiel ou pas. 
Céline énonce les fonctions essentielles de l'écrit :

- structurer sa pensée en se confrontant à soi-même (dialogue interne) et à la propre élaboration de sa pensée (extrait 3 )

- s'exprimer (extrait 1)

- transmettre, communiquer (extrait 2)

- garder des traces, archiver (extrait 4)

- construire des preuves de la réalité des choses, mais aussi, dans une perspective plus ontologique, des preuves d'existence (extrait 4)

\section{Extrait 4}

Enquêteur : Et ça t'embêterait si finalement en cours on ne faisait que parler si on n'écrivait pas

Céline : Moi ça m'embêterait ben disons qu'il y a besoin d'avoir des traces quoi ne serait-ce que dans un an j'ai besoin de ce que l'on a dit sur la vie d'Apollinaire je ne $m$ 'en rappellerai pas forcément [...] si on doit réviser que je n'ai pas de traces écrites je ne vois même pas ce qu'il faut réviser [...] et si je n'avais pas à réviser je pense j'écrirais quand même parce que dans la vie quotidienne dès que je pense à quelque chose pour ne pas l'oublier je l'écris que ce soit pour faire les courses ou que ce soit des pensées c'est pareil quoi [...] il me faut une trace [...] pour moi c'est une preuve Enquêteur: Une preuve que les choses ont eu lieu

Céline : Oui qu'on a bien existé

Ainsi le discours de Céline construit-il un rapport à l'écrit abouti qui n'édulcore pas la complexité de l'activité d'écriture. On peut penser que ce rapport positif et élaboré devrait constituer une aide aux apprentissages scolaires : apprentissage de l'écriture et apprentissages disciplinaires, acquisition de connaissances. Qu'en est-il exactement?

Pour analyser comment ce rapport à l'écrit s'investit dans l'écriture scolaire, nous mettrons en rapport ce discours sur l'écrit et l'écriture en général et les discours sur les écrits disciplinaires, et plus particulièrement les discours de Céline concernant les écrits spécifiques à trois disciplines : la synthèse à partir de documents en histoire, l'écriture d'invention en français, la rédaction de la solution à un problème en mathématique, ainsi qu'avec l'activité d'écriture lors d'un atelier d'écriture.

Pour chacune de ces disciplines, nous analyserons dans le discours de Céline

- comment elle décrit la norme ou la spécificité de l'écrit en question. L'indice linguistique pris en compte dans le discours sont les formules injonctives.

- comment s'exprime son rapport à l'écrit dans la tâche d'écriture en utilisant les indicateurs cités précédemment : investissement, opinions / sentiments / valeurs, et conceptions.

- comment elle décrit la réussite de cette activité d'écriture. Cette appréciation de sa performance est un des indices du rapport construit par le scripteur entre sa conception de l'écriture et sa réalisation (action).

\section{Céline et l'écriture de synthèse en histoire}

En histoire, une des démarches préconisées est l'étude de cas à partir d'un dossier documentaire qui « pose un problème et appelle une réponse » (MEN, 2007 : 48). Pour identifier le problème et élaborer la réponse, on attend des élèves qu'ils opèrent « l'exploitation analytique et synthétique des informations repérées dans les documents » (ibid.) Ainsi « la trace écrite résultant de l'étude de cas est[-elle] 
un tout : le dossier documentaire et le fruit de son exploitation (par exemple, un tableau et une synthèse sous une forme rédigée ou un schéma), ainsi que les notes prises lors de la mise en perspective. » (ibid: 49). Dans cette démarche l'écriture d'une synthèse apparaît comme un outil typique de l'élaboration de connaissances historiques.

\section{Extrait 6}

Céline : En histoire quand on va faire un commentaire de documents étant donné que j'ai du mal à cerner l'essentiel et que je me perds beaucoup dans les détails et que j'ai du mal à me détacher des documents je trouve cela plus dur mais c'est quand même intéressant mais je sais moins par où commencer quoi $[\ldots]$

Enquêteur: tu disais donc les documents d'histoire géo ça te paraît faire des synthèses c'est plus compliqué

Céline : Oui disons que c'est plus compliqué parce que déjà par exemple il faudrait faire un plan moi j'ai du mal à synthétiser tous les détails pour moi sont importants donc je n'arrive pas à me dire si j'oublie ça c'est peut-être important je vais passer à côté de quelque chose et puis en même temps je ne vois pas l'essentiel je ne vois pas ce qui est enfin j'ai du mal à hiérarchiser en fait pour le moment bon ça va quand même un peu mieux qu'en seconde mais ce n'est pas ça du tout quoi encore

\section{Identification de la norme}

Le discours de Céline indique qu'elle a identifié la norme qui régit l'exercice de la synthèse de documents en histoire et qu'elle est en capacité de la décrire. Elle formule explicitement qu'« il faudrait faire un plan » et quand elle décrit sa propre manière de réaliser la tâche, elle formule en creux les normes de cet écrit : cerner l'essentiel, hiérarchiser, synthétiser, se détacher des documents.

\section{Rapport à l'écrit et appropriation personnelle}

En disant que « c'est quand même intéressant », Céline exprime un certain investissement dans cette tâche. Cependant, elle signale aussi ses difficultés : "c'est dur ». Certaines de ces difficultés sont relatives aux opérations liées à la tâche de lecture pour appréhender le document : «j'ai du mal à cerner l'essentiel », « je me perds dans les détails », "je ne sais pas par où commencer», "j'ai du mal à synthétiser », " j'ai du mal à hiérarchiser », c'est-à-dire les opérations de repérage, tri, hiérarchisation, synthèse de l'information.

Céline explique aussi son attitude : «j'ai du mal à me détacher des documents » que l'on peut mettre en rapport avec son sentiment de crainte d'oublier quelque chose : « je n'arrive pas à me dire si j'oublie ça c'est peut-être important je vais passer à côté de quelque chose ». Cette attitude et ce sentiment correspondent à la conception très forte qu'elle a développée concernant son rapport à l'écrit personnel : écrire revient à garder des traces et des preuves que quelque chose existe (extrait 4 du rapport personnel). Il en résulte une certaine tension entre cette conception personnelle de l'écriture : garder des preuves et des traces, et celle que soustend l'écrit de synthèse : trier et structurer, donc perdre le détail pour garder l'essentiel.

\section{Appréciation - évaluation}

Parallèlement, si Céline décrit les difficultés de réalisation de la tâche, elle n'explique pas comment elle résout la difficulté rencontrée. Ceci ne signifie pas forcément qu'il n'y a pas de solution mise en œuvre, d'autant qu'elle signale : «pour le moment bon ça va quand même un peu mieux qu'en seconde mais ce n'est pas ça du tout quoi encore ». Mais selon elle, elle ne réussit pas encore correcte- 
ment cette tâche décriture et on peut supposer que le fait qu'elle ne verbalise pas ce qu'elle met en œuvre empêche la prise de conscience et en partie l'apprentissage.

\section{Conclusion}

Concernant cette tache de commentaire et synthèse de documents en histoire, on retient donc une tension entre la conception personnelle de l'écrit : garder des traces et construire des preuves, et la conception que sous-tend l'écrit disciplinaire : éliminer pour synthétiser et structurer. On remarque que Céline a du mal à choisir entre ces deux conceptions en jeu, d'où la difficulté à réaliser la tâche. On peut aussi remarquer qu'étrangement la conception «écrire pour structurer sa pensée » formulée pour l'écriture personnelle n'est pas activée pour résoudre la difficulté rencontrée. Ainsi Céline ne construit-elle pas de lien entre sa conception personnelle et la tâche à réaliser et n'investit pas une partie de son rapport personnel à l'écrit dans la tâche scolaire. On peut de ce fait faire l'hypothèse que les différents éléments de ce rapport à l'écrit n'agissent pas avec la même force au moment de la réalisation de la tâche.

\section{Céline et l'écriture d'invention en français}

Dans les classes de lycée, les élèves s'entraînent à des tâches dites d'écriture d'invention qui constitue aussi un des exercices du baccalauréat. La démarche de l'écriture d'invention a pour « but d'amener les élèves à la maîtrise de l'expression écrite autonome » $(\mathrm{MEN}, 2006)$. Elle se réalise « en liaison notamment avec les différents genres et registres étudiés» ( $i b i d)$. Ainsi, «lecture et écriture sont-elles associées dans des travaux de réécriture qui contribuent à une meilleure compréhension des textes » (ibid.). La démarche vise également à faire " apparaître les liens entre invention et argumentation » (ibid.)

\section{Extrait 7}

Céline : Dans l'écrit d'invention ce qui est bien c'est qu'en fait c'est comme si donc il y avait un carré à l'intérieur on fait ce qu'on veut mais il ne faut pas en sortir quoi donc moi comme je disais c'est écrire suivant enfin c'est inventer suivant un modèle donc d'un côté il y a des contraintes à respecter donc c'est à soi de les cerner de voir les limites mais en quoi on se confronte à soi même justement il y a un sujet donné c'est ce qu'on pense par rapport à ce sujet essayer ne pas être hors sujet se poser des questions voilà quoi je pense après chacun sa méthode

Enquêteur : Et c'est quoi ta méthode à toi alors

Céline : Je ne sais pas je vois l'énoncé donc je lis l'énoncé je me dis bon qu'est-ce qu'on me demande et je en général je suis assez lente donc je prends mon temps avant de me lancer je oui je relis enfin il faut que je sois sûre de mon coup avant de me lancer si j'ai un doute cela me bloque je me dis je vais partir là-dedans mais cela peut être hors sujet donc il faut que je sache un peu où je veux aller où je vais Enquêteur: Donc tu penses beaucoup avant d'écrire

Céline : Je pense beaucoup c'est un grand mot au début il faut juste le fil conducteur il faut mon cadre quoi après dedans une fois je réfléchis juste à mon cadre en fait ne pas sortir de là-dedans c'est tout une fois que j'ai trouvé le cadre après voilà et ça peut venir spontanément [...]

Céline : Par exemple des fois ça va et là par exemple on a eu un DM en français il fallait bon il y avait trois textes du $18^{\circ}$ siècle en fait il fallait faire un écrit d'invention par rapport à l'article de l'Encyclopédie [...] la prof nous a dit de réactualiser et moi je restais bloquée dans le $18^{\circ}$ donc j'ai eu du mal à m'en détacher donc je me suis dit bon voilà donc je me suis dit vu que j'ai du mal à m'en détacher je commence à partir du $18^{\circ}$ je fais une évolution et je pars sur le $21^{\circ}$ 


\section{Identification de la norme}

A la différence de l'exercice de la synthèse en histoire, concernant l'écriture d'invention, Céline décrit les normes qui régissent cette tâche avec précision : «il ne faut pas sortir (du carré) », "c'est inventer suivant un modèle », " il y a des contraintes à respecter, à cerner, ", «c'est voir les limites », "c'est ce qu'on pense par rapport à un sujet», «ne pas être hors sujet», c'est « se poser des questions ». Les formules sont précises et usent des différentes modalités de l'injonction.

\section{Rapport à l'écrit et appropriation personnelle}

Le développement aisé du discours et l'importance des formules positives ( «c'est bien ») suggèrent un investissement important dans cette tâche d'écriture. Cependant, en même temps, Céline exprime un sentiment d'inquiétude et d'insécurité : «je prends mon temps avant de me lancer», «je relis », « il faut que je sois sûre de mon coup avant de me lancer si j'ai un doute cela me bloque je me dis je vais partir là-dedans mais cela peut être hors sujet donc il faut que je sache un peu où je veux aller où je vais ». Mais cette insécurité semble compensée par la confiance que l'élève a en ses dispositions personnelles : «ça peut venir spontanément».

On remarque que, selon Céline, la conception de l'écriture qui sous-tend l'écriture d'invention : " on se confronte à soi-même », " on dit ce qu'on pense par rapport au sujet donné », " on se pose des questions ", est conforme à sa conception personnelle : écrire pour s'exprimer (extrait $1 \mathrm{du}$ rapport personnel). Ceci peut paraître paradoxal dans le mesure où dans l'écriture d'invention, et Céline a bien identifié cette spécificité, il s'agit d'abord de répondre à une contrainte. Or, quand elle exprime son attitude et sa conception de cette tâche, elle retient d'abord l'idée de liberté. Le paradoxe s'accroit quand elle exprime pour l'écriture d'invention la même difficulté à se détacher du modèle. Cette difficulté a déjà été formulée à propos de l'exercice de synthèse en histoire. On peut la relier au sentiment de peur de perdre quelque chose exprimé dans la conception personnelle de l'écrit comme une trace et une preuve.

\section{Appréciation - évaluation}

L'explicitation de la gestion de la tâche aboutit à la verbalisation très précise de la manière de résoudre les difficultés et les opérations de sa mise en œuvre : lire et relire l'énoncé, identifier la demande, déterminer un cadre et un fil conducteur, réfléchir et prévoir avant d'écrire. Céline est également capable de décrire sa stratégie et la progression de son raisonnement : « je me suis dit vu que j'ai du mal à m'en détacher je commence à partir du $18^{\circ} \mathrm{je}$ fais une évolution et je pars sur 1 e $21^{\circ} \gg$. Au bout du compte, elle parvient à réussir la tâche et semble se sentir plutôt à l'aise dans l'écriture d'invention.

\section{Conclusion}

Ce qui caractérise la conception de Céline de l'écriture d'invention est donc le paradoxe. En effet, alors qu'il faut réaliser une tâche à forte contrainte, elle retient l'idée de liberté que laisse cette tâche. Ce faisant, elle met en conformité la norme de l'exercice scolaire avec sa conception personnelle de l'écriture et s'approprie l'exercice et sa norme. Ainsi la conception de l'écriture comme expression de soi l'emporte-t-elle sur la conception qui consiste à considérer qu'écrire c'est respecter des contraintes. Conception d'ailleurs que Céline n'exprime pas quand elle parle de son rapport personnel à l'écrit. On peut alors faire l'hypothèse que la diffi- 
culté à se détacher du modèle est cette fois, et à la différence de ce qui se passe pour l'exercice de la synthèse en histoire, surmontée et compensée par la motivation à l'expression de soi (liberté). Cette conception prend ainsi le dessus sur les autres conceptions, et permet de surmonter les sentiments d'insécurité et plus particulièrement le sentiment de perdre quelque chose. Ainsi l'écriture d'invention permetelle l'actualisation de la conception s'exprimer et structurer la pensée, qui l'emporte sur la conception garder la trace de. C'est cette conception qui permet de surmonter les difficultés rencontrées par la norme de l'exercice scolaire.

\title{
5. Céline et la rédaction de la solution d'un problème en mathématiques
}

Les programmes de mathématique pour les classes de lycée insistent sur la diversité des activités à réaliser, parmi lesquelles « raisonner, démontrer, trouver des résultats partiels et les mettre en perspective » et « expliquer oralement une démarche, communiquer un résultat par oral ou par écrit » (MEN, 2009 : 2). Ainsi, une part importante des tâches d'écriture réalisées en mathématique consiste-telle à rédiger la solution d'un problème. Dans l'entretien qu'elle nous accorde, et malgré les relances de l'enquêteur, Céline développe peu son discours à propos des cours de mathématique. Cependant, elle ne formule pas un discours de rejet des mathématiques.

\begin{abstract}
Extrait 8
Enquêteur : Et en maths est-ce que vous avez ce travail méthodologique aussi sur comment écrire une réponse à un problème ou sur l'écriture d'une solution ou pas Céline : Ben disons que non en maths on fait une phrase vite fait pour justifier comme quoi voilà en même temps ce n'est pas non plus [...]

Enquêteur: Oui tu n'as pas l'impression qu'il y a la même exigence au niveau de la rédaction en maths qu'en français

Céline : Non parce que c'est des maths mais ça n'empêche pas qu'il faut justifier et qu'il faut faire des phrases avec des majuscules et un point et des verbes et des compléments mais mais bon on ne va pas non plus on a justifié c'est une phrase complète c'est tout quoi

Céline : En français on ne nous demande pas non plus d'écrire un poème pour rédiger mais ce n'est pas pareil la place des mots vraiment en maths elle est moins importante c'est les calculs qui voilà ce n'est pas pareil
\end{abstract}

\section{Identification de la norme}

Quand l'enquêteur interroge Céline sur ce qu'il faut faire en écriture en mathématique, celle-ci insiste sur les aspects linguistiques et codiques de l'énoncé à produire : « en maths on fait une phrase vite fait pour justifier » et « il faut faire des phrases avec des majuscules et un point et des verbes et des compléments mais bon on ne va pas non plus on a justifié c'est une phrase complète c'est tout quoi ». L'énoncé à produire se trouve ainsi réduit à sa dimension grammaticale, alors que sa visée pragmatique (justifier) est reléguée au second plan. Ainsi Céline identifie-t-elle une norme, mais, à part le fait qu'il faille justifier le résultat, elle formule en matière d'écriture surtout des stéréotypes, allant presque jusqu'à nier qu'il puisse y avoir une spécificité de l'écriture mathématique.

\section{Rapport à l'écrit et appropriation personnelle}

Par ailleurs, dans cet extrait de l'entretien concernant les mathématiques, Céline n'exprime aucun investissement, ni aucun sentiment. Ainsi la rapport à l'écri- 
ture mathématique exprimé ici est-il distancié, voire désinvesti. Le discours développe une conception de l'écrit qui ne l'envisage que comme forme syntaxique et pour remplir une seule fonction : justifier. Cette première conception de l'écriture envisagée essentiellement dans sa dimension syntaxique n'apparaît pas dans la conception de l'écriture personnelle. En revanche, on peut rapprocher cette fonction «justifier» de la conception de l'écrit comme preuve exprimée dans le rapport à l'écrit personnel, en considérant que l'écrit mathématique fournit une preuve de la justesse du raisonnement. Mais Céline ne développe pas cette conception. Cela suggère qu'elle établit une différence entre la dimension ontologique de l'écriture (écrire est une preuve que quelque chose ou quelqu'un existe) et sa dimension intellectuelle (écrire fournit la preuve de la justesse d'un raisonnement).

Il faut aussi remarquer que Céline n'active pas sa conception personnelle de l'écriture comme outil de structuration de la pensée (extrait 1 du rapport personnel), alors que cette conception constitue un aspect majeur de l'écriture mathématique, discipline dans laquelle écrire permet la construction du raisonnement mathématique, et dont on pourrait s'attendre qu'elle soit actualisée par l'élève au cours de cette activité de résolution de problème.

\section{Appréciation - évaluation}

A propos de l'activité d'écriture en mathématique, Céline n'indique pas comment elle s'approprie la tâche et la résout. Elle ne dit pas non plus si elle réussit ou pas. Cette tâche d'écriture génère une verbalisation moins importante que pour les autres disciplines et la locutrice ne semble pas éprouver le besoin de développer son propos. Cette attitude est en conformité avec la réduction de cette activité à une simple mise en phrases.

\section{Conclusion}

Ainsi le discours de Céline construit-il une disjonction radicale entre sa conception personnelle de l'écriture, son rapport personnel à l'écrit et l'écriture mathématique. Comme si ce qui s'écrivait en mathématique n'était pas de l'écriture

\section{Céline et l'écriture à contrainte en atelier d'écriture}

Le projet pédagogique du lycée des décrocheurs inclut un atelier d'écriture intitulé « la boutique d'écriture », et qui se déroule une fois par semaine pendant une heure. Il est animé par un professeur de n'importe quelle discipline. Au cours de cet atelier, les élèves ont un texte à écrire à partir d'une consigne qui indique le type de contrainte auquel il doit répondre : générique, stylistique, thématique, lexicale, etc. Une même consigne peut combiner plusieurs contraintes.

\section{Extrait 9}

Céline : [la boutique d'écriture] c'est partir du principe que la contrainte stimule l'imaginaire

Enquêteur: Alors cela se passe comment la boutique d'écriture

Céline : ça se passe comment déjà ça passe vite et chaque semaine on a une contrainte oui voilà en général on a le choix entre plusieurs sujets on choisit celui qui nous tente le plus chacun écrit son petit truc et puis après à la fin ceux qui veulent lire ils lisent ils en font profiter les autres voilà et donc oui c'est bien

Céline : Je trouve ça intéressant déjà d'écouter ce que les autres ont écrit parce qu'il y a vraiment des systèmes différents et puis la contrainte moi à la base j'aime bien quand il n'y a pas de contraintes pour écrire mais en fait non c'est vrai que c'est intéressant quand même d'écrire sous contrainte 
Céline : C'est vrai que ça permet d'un côté avec une contrainte au moins on a une base on part de là et puis on a l'impression que c'est une limite et en fait cela nous permet de au delà de la limite il y a d'autres choses voilà

Enquêteur: Et toi tu entres bien dans cette démarche

Céline : J'essaie hein je rentre bien en tout cas moi j'aime bien ça me plaît donc même si des fois il y a des sujets qui inspirent plus que d'autres même si ça ne m'inspire pas trop j'essaie quand même de faire un petit truc même si ce n'est pas terrible juste histoire de dire d'avoir fait quelque chose d'avoir dit ce que je pensais quoi Enquêteur: Oui et quand tu dis je fais un petit truc pas terrible qu'est-ce que tu entends par pas terrible pourquoi tu considères que ce n'est pas terrible

Céline : Parce que je ne suis pas contente de je me dis cela ne parle pas tant que ça quoi après de toute façon la boutique d'écriture on n'est pas là pour faire des super œuvres donc mais c'est déjà bien si au moins on arrive à être un peu content de ce que l'on a fait et si ça plaît aux autres ça c'est autre chose mais bon si on a écrit quelque chose et si on se dit ça ne m'a pas inspiré ce n'est pas quelque chose qui va me marquer voilà

Enquêteur : D'accord et quand tu es contente qu'est-ce qui fait que tu es contente qu'est-ce que c'est pour toi quand c'est réussi ou que tu es contente

Céline : Quand j'ai réussi à exprimer ce que je ressentais quand j'ai réussi à mettre par écrit à peu près j'ai pu me rapprocher de ce qu'il y avait au fond de moi ce que j'avais envie de dire aux autres

\section{Identification de la norme}

Encore une fois, le discours de Céline montre qu'elle identifie les normes à l'œuvre au niveau de l'écrit à produire. Celles-ci appartiennent à plusieurs registres : le principe même de l'écriture à contrainte : «c'est partir du principe que la contrainte stimule l'imaginaire »; les modalités de la pratique d'écriture proposée au sein de la boutique d'écriture : fréquence «ça se passe chaque semaine », intensité « ça se passe vite»; mais aussi les modalités de la mise en œuvre de l'activité d'écriture : choisir un sujet, écrire, lire, écouter ; le niveau d'exigence : « on n'est pas là pour faire de super œuvres ».

\section{Rapport à l'écrit et appropriation personnelle}

On remarque que l'évocation de la boutique d'écriture entraine un discours abondant et évaluatif, qui insiste sur l'investissement. Celui-ci est construit dans deux registres : d'une part affectif : «c'est bien », «j'aime bien », « ça me plait», « je ne suis pas contente », « on arrive à être un peu contente»; d'autre part cognitif : «c'est intéressant d'écouter ce que les autres ont écrit » pour découvrir différents systèmes d'écriture, «c'est intéressant d'écrire sous contrainte ».

Cependant cet investissement est immédiatement euphémisé : « chacun écrit son petit truc », "si on se dit ça ne m'a pas inspiré ce n'est pas quelque chose qui va me marquer ». Ainsi Céline exprime-t-elle des sentiments positifs, et cette fois aucune anxiété, mais en même temps seulement du contentement ou même du détachement. Au bout du compte, il s'agit d'un investissement maitrisé ou mis à distance. Dans le même ordre d'idée, le texte produit n'est pas valorisé.

La conception de l'écrit qui sous-tend cette tâche articule l'expression de soi et la transmission du texte produit : « [je suis contente] quand j'ai réussi à exprimer ce que je ressentais quand j'ai réussi à mettre par écrit à peu près j'ai pu me rapprocher de ce qu'il y avait au fond de moi ce que j'avais envie de dire aux autres ». Cette conception est donc en conformité avec la conception personnelle de l'écriture et le rapport personnel à l'écrit (extraits 1 et 2 du rapport personnel). 
Parallèlement, le discours de Céline construit un rapport dialectique entre la contrainte et ses effets bénéfiques dans la réalisation de la tâche, qui finalement, alors que Céline affirme qu'elle aime la liberté dans l'écriture, aboutit à la valorisation de la norme et de la contrainte : «moi à la base j'aime bien quand il n'y a pas de contraintes pour écrire mais en fait non c'est vrai que c'est intéressant quand même d'écrire sous contrainte [...] c'est vrai que ça permet d'un côté avec une contrainte au moins on a une base on part de là et puis on a l'impression que c'est une limite et en fait cela nous permet de au delà de la limite il y a d'autres choses »

On le voit, cette activité d'écriture en atelier est en forte résonance avec la conception de l'écriture personnelle de Céline et son rapport personnel à l'écrit.

\section{Appréciation - évaluation}

Et pourtant le discours développe peu l'explicitation de la tâche : « j'essaie quand même de faire un petit truc même si ce n'est pas terrible juste histoire de dire d'avoir fait quelque chose ». Et finalement la réussite de Céline semble, du moins à ses yeux, toute relative.

\section{Conclusion}

Quand il s'agit des tâches d'écriture au sein d'un atelier, on peut donc remarquer que malgré un discours valorisant, la conformité entre la conception personnelle de l'écriture et celle qui sous-tend la norme à l'œuvre dans la tâche, l'investissement de Céline et la réussite sont relatifs. Ce qui peut sembler contradictoire. En même temps, c'est pour cette tâche que Céline développe le discours le plus dialectique comme si elle était à la recherche d'un consensus entre sa conception personnelle de l'écriture: "j'aime bien quand il n'y a pas de contrainte » et la norme forte de l'écriture sous contrainte.

\section{Conclusion.}

\section{Rapport aux écrits disciplinaires : conflits et réussite}

Notons tout d'abord qu'en dépit de la diversité des formes d'écrits selon les disciplines et des normes à respecter, le discours de Céline montre qu'à chaque fois elle parvient à en identifier les principaux aspects, sauf en mathématique où elle s'en tient à une description très stéréotypée de ce qu'est, selon elle, l'écriture mathématique.

A chaque fois aussi, selon chaque discipline, le discours de Céline construit des rapports différents entre la conception personnelle de l'écriture et le rapport personnel à l'écrit d'une part, et l'activation de ce rapport à et de cette conception dans la réalisation de la tâche d'écriture spécifique de la discipline d'autre part. Ainsi à chaque fois, la prise en compte par l'élève de la tâche d'écriture scolaire et des normes textuelles et procédurales qu'elle impose établit-elle un conflit entre les conceptions personnelles de l'écriture et celles qui sous-tendent la tâche scolaire d'écriture. On a pu identifier quatre sortes de conflit : la tension, le paradoxe, la disjonction et la contradiction. Or pour réaliser la tâche d'écriture et la réussir l'élève doit surmonter le conflit. Plusieurs manières sont à l'œuvre.

En histoire, la norme imposée par l'écrit de synthèse provoque une tension entre deux conceptions, mais on remarque que l'élève n'active pas vraiment sa conception personnelle et son rapport à l'écrit pour résoudre le conflit. Au final, la tâche d'écriture n'est pas vraiment réussie.

En français, l'exercice d'invention aboutit à un paradoxe conceptuel, mais 
l'élève active sa conception personnelle (s'exprimer / structurer sa pensée) qui l'emporte sur celle imposée par la norme scolaire. Dans ce cas, la tâche est réussie.

En boutique d'écriture, au départ la norme imposée par l'écriture à contrainte est en contradiction avec la conception personnelle de l'élève. Mais celle-ci recherche un consensus qui aboutit à une réussite relative de la tâche.

En mathématique, la conception personnelle de l'élève et la norme scolaire de l'écriture mathématique sont en disjonction. Bien qu'il y ait des liens entre ces deux conceptions, l'élève ne les construit pas. Au final, la question de la réussite n'est pas évoquée.

Si on met plus nettement en rapport les différentes manières de résoudre le conflit et la réussite de la tâche, on remarque que :

- ne pas résoudre le conflit (écriture de synthèse) empêche une réelle réussite de la tâche,

- activer une conception personnelle qui l'emporte sur la conception imposée par la tâche (écriture d'invention) aboutit à la réussite,

- rechercher le consensus entre la conception personnelle et celle imposée par la tâche (écriture à contrainte) ne débouche pas forcément sur la réussite de la tâche,

- ne pas percevoir le conflit (écriture mathématique) empêche de percevoir les fonctions de l'écriture dans la tâche à réaliser, ce qui semble empêcher aussi une réflexion méta cognitive et méta scripturale, et une perception de ce qui est réussi ou pas.

Concernant le cas qui nous occupe, il apparaît, et l'on s'y attendait, que le conflit conceptuel entre l'écriture personnelle et l'écriture scolaire rend la réussite de la tâche d'écriture hasardeuse (écriture de synthèse en histoire, écriture de résolution de problème en mathématique)

En revanche, et c'est plus étonnant, on voit que quand l'élève parvient à mettre en adéquation sa conception personnelle et celle imposée par la norme scolaire, il n'y a pas forcément de réussite (écriture à contrainte). Ainsi l'adéquation entre les conceptions personnelle et scolaire de l'écriture ne semble-t-elle pas être une garantie de réussite.

On remarque aussi que l'adéquation entre la conception personnelle et la conception scolaire n'aboutit pas non plus forcément à un investissement important de l'élève dans la tâche (boutique d'écriture).

Faut-il en déduire que la notion de rapport à l'écrit ne serait pas opérationnelle ? Nous ne le croyons pas. Au contraire, nous pensons plutôt que cette étude de cas met en évidence qu'elle doit être mieux intégrée à la tâche d'écriture et à son apprentissage. De cette manière les élèves pourraient, non seulement prendre conscience de leurs conceptions personnelles de l'écriture, mais aussi des écarts avec celles sous-tendues par les tâches d'écriture dans les différentes disciplines. Cela peut constituer pour eux un moyen de mieux mesurer l'investissement nécessaire et les stratégies intellectuelles efficaces pour réduire les éventuels conflits entre leur conception personnelle de l'écriture et les conceptions scolaires. 


\section{Bibliographie}

B AKHTINE, M. (1984) : Esthétique de la création verbale, traduit par Alfreda Aucouturier, Paris : NRF-Gallimard.

BARRÉ-DE MINIAC, C. (2000) : Le rapport à l'écriture : aspects théoriques et didactiques. Villeneuve-d'Ascq : Septentrion.

— (2002) : «Le rapport à l'écriture. Une notion à plusieurs dimensions », Pratiques $\mathrm{n}^{\circ} 113 / 114$, pp. 29-39.

BARRÉ-DE MINIAC, C., \& REUTER, Y. (2006) : Apprendre à écrire au collège dans les différentes disciplines, Paris : INRP, « Documents et travaux de recherche en éducation $»$.

ChARlot, B., BAUtier, E. \& RocheX, Y. (1992) : École et savoir dans les banlieues... et ailleurs, Paris : Armand Colin.

CHARLOT, B. (1999) : Le rapport au savoir en milieu populaire. Une recherche dans les lycées professionnels de banlieue, Paris : Anthropos.

CHARTRAND, S-G., (dir.) (2008) : Le rapport à l'écrit : un outil pour enseigner de l'école à l'université, Namur : Presses Universitaires de Namur / Cedocef.

Delamotte, R., Gippet, F., Jorro, A., Penloup, M.-C. (2000) : Passages à l'écriture, un défi pour les apprenants et pour les formateurs, Paris : PUF.

DELCAMBRE, I. \& REUTER, Y., (coord.) (2002) : Images du scripteur et rapport àl'écriture, Pratiques, $\mathrm{n}^{\circ} 113-114$, Metz : CRESEF.

Halté, J.-F., Petitjean, A. \& Plane, S., (coord.) (2002) : L'écriture et son apprentissage, Pratiques $\mathrm{n}^{\circ} 115-116$, Metz : CRESEF.

INRP (1999) : Production d'écrits et construction des savoirs dans les différentes disciplines scolaires : gestion de l'hétérogénéité des élèves.

LAHANIER-REUTER, D. \& REUTER, Y. (2006) : «Discours de collégiens sur l'écriture et les disciplines scolaires », Barré-De Miniac, C., \& Reuter, Y., 2006, Apprendre à écrire au collège dans les différentes disciplines, Paris : INRP, « Documents et travaux de recherche en éducation ", pp. 23-58.

LAHIRE, B. (1993) : Culture écrite et inégalités scolaires. Sociologie de l'«échec » scolaire à l'école primaire, Lyon : Presses Universitaires de Lyon. - (1995) : Tableaux de famille, Paris : Gallimard, Ed. Du Seuil.

MinistÈRE de l'ÉDUCATION NATIONALE (2006) : Programme d'enseignement de français en classe de première des séries générales et technologiques, Journal Officiel du 18-10-2006.

- (2007) : Documents d'accompagnement aux programmes d'histoire, géographie, classes de seconde, première terminale, voie générale, CNDP.

- (2009) : Programme de mathématiques pour la classe de seconde, CNDP. 